\title{
Surveillance of pyrazinamide susceptibility among multidrug-resistant Mycobacterium tuberculosis isolates from Siriraj Hospital, Thailand
}

\author{
Jirarut Jonmalung ${ }^{1}$, Therdsak Prammananan ${ }^{2,4}$, Manoon Leechawengwongs ${ }^{3,4}$, Angkana Chaiprasert $^{1,4^{*}}$
}

\begin{abstract}
Background: Susceptibility testing of pyrazinamide (PZA) against Mycobacterium tuberculosis is difficult to perform because the acidity of culture medium that is required for drug activity also inhibits the growth of bacteria. In Thailand, very limited information has been generated on PZA resistance, particularly among multidrug-resistant tuberculosis (MDR-TB) isolated from Thailand. Only two studies on PZA susceptibility among Thai M. tuberculosis strains have been reported; one used a pyrazinamidase assay, and the other used the BACTEC 460 TB for PZA susceptibility testing. In this study, we determined the percentage of strains possessing pyrazinamide resistance among pan-susceptible M. tuberculosis and MDR-TB isolates by using the pyrazinamidase assay, BACTEC MGIT 960 PZA method and pncA sequencing, and assessed the correlation in the data generated using these methods. The type and frequency of mutations in pncA were also determined.
\end{abstract}

Results: Overall, 150 M. tuberculosis isolates, consisting of 50 susceptible and 100 MDR-TB isolates, were tested for PZA susceptibility by BACTEC MGIT 960 PZA, the pyrazinamidase assay and pncA sequencing. The study indicated PZA resistance in $6 \%$ and $49 \%$ of susceptible and MDR-TB isolates, respectively. In comparison to the BACTEC MGIT 960 PZA, the PZase assay showed $65.4 \%$ sensitivity and $100 \%$ specificity, whereas pncA sequencing showed $75 \%$ sensitivity and $89.8 \%$ specificity. Twenty-four mutation types were found in this study, with the most frequent mutation (16\%) being His71Asp. Of these mutations, eight have not been previously described. The lle31Ser and lle31Thr mutations were found both in PZA susceptible and resistant isolates, suggesting that mutation of this codon might not play a role on PZA resistance.

Conclusions: Our findings suggest that phenotypic susceptibility testing is still essential for the detection of PZA resistance, especially for MDR-TB isolates. Some mutations were not associated with resistance and could lead to misinterpretation of the genotypic methods. This information could be helpful for clinicians in managing tuberculosis patients and frequencies, and the types of pncA mutations should offer baseline information on PZA resistance.

\section{Background}

Tuberculosis (TB) is a public health problem caused by Mycobacterium tuberculosis. Thailand was ranked $18^{\text {th }}$ among high-burden countries, with 91,000 cases per year and new cases of MDR-TB (resistance to at least isoniazid and rifampicin) of approximately $1.7 \%$ [1]. Tuberculosis infection is increasing in human immunodeficiency virus (HIV) co-infected patients, affecting the

\footnotetext{
* Correspondence: siacp@mahidol.ac.th

'Department of Microbiology, Faculty of Medicine Siriraj Hospital, Mahidol University, Bangkok, 10700, Thailand

Full list of author information is available at the end of the article
}

TB control program as about one-third of Thai HIV/ AIDS patients present with active TB [2-5]. The standard regimen for the treatment of TB consists of 2 months of intensive treatment with isoniazid, rifampicin, ethambutol, and pyrazinamide (H, R, E, and Z), followed by 4 months of maintenance treatment with isoniazid and rifampicin ( $\mathrm{H}$ and $\mathrm{R})$. Whereas other first-line drugs do not reveal any problem for susceptibility testing, this is not true for pyrazinamide, as it is active against tubercle bacilli only at an acidic $\mathrm{pH}$ (e.g., $\mathrm{pH}$ 5.5), resulting in that it cannot use conventional culture medium for susceptibility testing [6].

\section{Ciomed Central}


Pyrazinamide (PZA, $\mathrm{Z}$ ) is a prodrug that requires conversion to the active form, pyrazinoic acid (POA), by mycobacterial pyrazinamidase (PZase) [7]. The exact target of POA is unknown. It has been suggested that the accumulation of POA in acidic conditions (from lactic acid produced by inflammation cells) leads to acidification of the cytoplasm and subsequent cell damage $[7,8]$. Mycobacterial pyrazinamidase is encoded by $p n c A$, and mutations in this gene have been demonstrated as the major mechanism of PZA resistance [9]. Several mutations, including missense, insertion, deletion and nonsense mutations, have been reported and located in both the putative promoter and coding regions of $p n c A$ [10]. PZA-resistant $M$. tuberculosis strains are usually correlated with defective PZase activity, but some PZA resistant strains have been reported to contain wild-type $p n c A$ and to maintain PZase activity [11-14], suggesting that other unknown resistance mechanisms could be responsible for the resistance phenotype.

PZA susceptibility testing is difficult because the acidity of culture medium needed for drug activity also restricts the growth of $M$. tuberculosis. The use of large inoculum sizes results in the release of $\mathrm{NH}_{3}$, leading to increased $\mathrm{pH}$ and inactivated PZA [7]. The BACTEC $460 \mathrm{~TB}$ radiometric method has been validated and developed as the reference method for PZA susceptibility testing [15]. Recently, PZA susceptibility testing has been performed by the nonradiometric, fully automated, continuous-monitoring MGIT 960 system (Becton Dickinson), which produced a rapid and reliable result $[16,17]$. Many studies revealed a good correlation between loss of PZase activity and resistance to PZA [18-22]. Thus, the detection of PZase activity has been used for PZA susceptibility testing. Nevertheless, various levels of sensitivity (79-96\%) of the PZase assay for PZA susceptibility testing have been reported [20-22]. In Thailand, only two studies on PZA susceptibility among Thai M. tuberculosis strains have been reported, and the results revealed that the initial PZA resistance was $5.95 \%$ and $7.8 \%$ when detected by the PZase assay [18] and by BACTEC $460 \mathrm{~TB}$ [23], respectively. In this study, we determined the percentage of strains that exhibited pyrazinamide resistance among pan-susceptible $M$. tuberculosis and MDR-TB isolates by using the pyrazinamidase assay, BACTEC MGIT 960 PZA method and $p n c A$ sequencing, and we evaluated the correlation of the results obtained with these methods. $p n c A$ mutation type and frequency were also evaluated.

\section{Methods}

\section{Mycobacterial isolates}

During 2005-2007, there were 4,536 M. tuberculosis isolates from 7,807 sputum samples sending from all parts of Thailand (118 hospitals and 43 of 76 provinces) to the Molecular Mycology and Mycobacteriology Laboratory, Drug-Resistant Tuberculosis Research Fund, Department of Microbiology, Faculty of Medicine Siriraj Hospital, Mahidol University. Of these, 220 and 4,316 isolates were identified as MDR$\mathrm{TB}$ and non MDR-TB, including pan-susceptible isolates respectively. One hundred and fifty $M$. tuberculosis clinical isolates, consisting of 50 pan-susceptible isolates (susceptible to isoniazid, rifampicin, ethambutol, and streptomycin) and 100 isolates of MDR-TB, were selected based on their ability to re-cultivate from stock cultures and availability of demographic data. The MDR-TB isolates contain 17, 13, 26 and 44 isolates resisted to isoniazid and rifampicin, to isoniazid, rifampicin and streptomycin, to isoniazid, rifampicin and ethambutol and to all four drugs respectively. These isolates were identified to species using the inhouse one-tube multiplex PCR [24], and antimicrobial susceptibility testing to isoniazid, rifampicin, ethambutol and streptomycim was performed by the standard proportion method on M7H10 agar as recommended by the CDC [25] and NCCLS [15]. Each isolate obtained from individual patient. The patients include new and previously treated patients with the age ranged from 5 to 81 years and comprised of $65 \%$ male and $35 \%$ female. Only $21 \%$ were known human immunodeficiency virus (HIV) status. Among these, 52\% were HIV-positive.

\section{PZA susceptibility testing}

Pyrazinamide susceptibility testing was performed using the BACTEC MGIT 960 PZA system (Becton Dickinson) as recommended by the manufacturer. The medium used was modified Middlebrook 7H9 broth ( $\mathrm{pH}$ 5.9) containing $100 \mu \mathrm{g} / \mathrm{ml}$ PZA. Mycobacterium bovis BCG ATCC 34540 and Mycobacterium tuberculosis H37Rv ATCC 27294 were used as pyrazinamide resistant and susceptible controls, respectively. The control strains were included in all test sets.

\section{Pyrazinamidase assay}

Pyrazinamidase activity was determined by Wayne's method [26]. This method is based on the detection of POA, which forms a compound with ferrous ammonium sulphate to produce a brownish or pink colour. Briefly, a heavy loopful of $M$. tuberculosis colonies was obtained from cultures that were actively growing in LJ medium and inoculated onto the surfaces of two agar butt tubes, each containing $5 \mathrm{ml}$ of Wayne's medium supplemented with $100 \mu \mathrm{g} / \mathrm{ml}$ of PZA (Sigma-Aldrich, USA). The tubes were incubated at $37^{\circ} \mathrm{C}$. Four days after incubation, $1 \mathrm{ml}$ of freshly prepared $1 \%$ ferrous ammonium sulphate was added to the first tube. The tube was left at room temperature for 30 minutes and examined. The 
assay was positive if a pink or brownish band was present on the surface of the agar. If the test was negative, the test was repeated with a second tube and examined after 7 days of incubation. The results were blindly read by two independent observers. $M$. bovis BCG and $M$. tuberculosis $\mathrm{H} 37 \mathrm{Rv}$ were used as negative and positive controls, respectively.

\section{DNA extraction}

Mycobacterial DNAs were extracted by the boiling method [27]. Briefly, one loopful of $M$. tuberculosis colonies obtained from LJ medium was suspended in $200 \mu \mathrm{l}$ of TE buffer (10 mM Tris-HCl, $1 \mathrm{mM}$ EDTA, $\mathrm{pH}$ 8.0) and boiled for 20 minutes. The supernatant was collected by centrifugation at 12,000 rpm for $5 \mathrm{~min}$ and used as the DNA template for amplification.

Amplification and sequencing of the amplified pncA gene The $p n c A$ forward primer, pncAF1, (5'-GCGGCG TCATGGACCCTATATC-3') was located 82 bp upstream of the start codon, and the reverse primer, pncAR1, (5'-CTTGCGGCGAGCG CTCCA -3') was located 54 bp downstream of the stop codon of $M$. tuberculosis pncA (Rv2043c). The expected size of the PCR products was $696 \mathrm{bp}$. PCR was performed in a total volume of $50 \mu \mathrm{l}$, and the PCR reaction mixture consisted of $0.25 \mathrm{mM}$ dNTP (Fermentas, CA, USA), $10 \mathrm{mM}$ Tris- $\mathrm{HCl}$ (pH 8.3), $50 \mathrm{mM} \mathrm{KCl}, 2.0 \mathrm{mM} \mathrm{MgCl} 2$, 20 pmol of each primer, 1 unit of Taq DNA polymerase (Fermentas, CA, USA) and $5 \mu \mathrm{l}$ of crude DNA. The PCR reactions were performed under the following conditions: initial denaturation at $94^{\circ} \mathrm{C}$ for $5 \mathrm{~min} ; 40$ cycles of denaturation at $94^{\circ} \mathrm{C}$ for $1 \mathrm{~min}$, annealing at $62^{\circ} \mathrm{C}$ for $1 \mathrm{~min}$ and extension at $72^{\circ} \mathrm{C}$ for $1 \mathrm{~min}$; and 1 final cycle of extension at $72^{\circ} \mathrm{C}$ for $10 \mathrm{~min}$. PCR products were analysed by $1 \%$ agarose gel electrophoresis, and subsequently they were purified using the Gel Extraction Kit (Qiagen, Germany). Finally, purified DNAs were directly sequenced with the ABI PRISM 3730XL Analyzer, (Applied Biosystems, Foster City, USA) using the pncAF1 and pncAR1 primers as sequencing primers. The obtained sequences were compared with the sequence of $M$. tuberculosis $\mathrm{H} 37 \mathrm{Rv}$ pncA (Accession no. NC_000962) by using the blastn program http://blast. ncbi.nlm.nih.gov/Blast.cgi.

\section{Results}

Pyrazinamide susceptibility testing by the phenotypic method

MGIT 960 susceptibility testing demonstrated that 52 (34.6\%) of 150 isolates were phenotypically resistant to PZA. More specifically, 3 (6\%) of 50 pan-susceptible $M$. tuberculosis isolates were resistant to PZA, whereas 49 (49\%) of 100 MDR-TB isolates were PZA-resistant, as summarised in Table 1.

\section{Correlation of PZA susceptibility testing and the pyrazinamidase assay}

Pyrazinamidase activity was detected in all pan-susceptible isolates and in 3 PZA-resistant isolates. Among the 100 MDR-TB isolates, 85 provided concordant results between the two methods; 51 isolates with phenotypic susceptibility to PZA had PZase activity, whereas PZase activity could not be detected in 34 PZA-resistant isolates. However, $15 \mathrm{MDR}-\mathrm{TB}$ isolates with PZA-resistant phenotypes had PZase activity (Table 1). Compared to the BACTEC MGIT 960 PZA system, the PZase assay showed $65.4 \%$ sensitivity and $100 \%$ specificity.

\section{Correlation of PZA susceptibility, pyrazinamidase assay and mutations in pncA}

Susceptibility testing by BACTEC MGIT 960 PZA revealed 98 PZA-susceptible isolates with positive PZase activity. Of these, 88 isolates had no mutations in $p n c A$, whereas 10 isolates harboured mutations at nucleotide 92 ( $\mathrm{T} \rightarrow \mathrm{G} / \mathrm{C}$ ), causing an amino acid change from isoleucine to serine or threonine, respectively, at codon 31 . Thirty-two of the PZA-resistant isolates without PZase activity contained mutations in $p n c A$, with 18 types of nucleotide substitutions in the coding region, 2 mutational types in the putative promoter region, 2 nucleotide insertions, and one nonsense mutation, as summarised in Table 2. Interestingly, there were two PZA-resistant isolates with negative PZase activity that were mutated at codon 31 (Ile $\rightarrow$ Ser), a mutant that was also found in PZA-susceptible isolates. In contrast, five PZA-resistant isolates that had Ile31Ser or Ile31Thr mutations possessed PZase activity (Table 2). Moreover, there were $13(26.5 \%)$ isolates with wild-type $p n c A$ and PZase activity but possessed resistant phenotypes. Thus, the sensitivity and specificity of $p n c A$ sequencing were

Table 1 Comparison of pncA sequencing, the pyrazinamidase assay, and the MGIT 960 system for PZA susceptibility testing.

\begin{tabular}{|c|c|c|c|c|c|}
\hline $\begin{array}{l}\text { M. tb strains } \\
\text { (total no. of isolates) }\end{array}$ & $\begin{array}{l}\text { MGIT (S) } \\
\text { PZase (pos) } \\
\text { pncA (wt) }\end{array}$ & $\begin{array}{l}\text { MGIT (S) } \\
\text { PZase (pos) } \\
\text { pncA (mut) }\end{array}$ & $\begin{array}{l}\text { MGIT (R) } \\
\text { PZase (neg) } \\
\text { pncA (mut) }\end{array}$ & $\begin{array}{l}\text { MGIT (R) } \\
\text { PZase (pos) } \\
\text { pncA (wt) }\end{array}$ & $\begin{array}{l}\text { MGIT (R) } \\
\text { PZase (pos) } \\
\text { pncA (mut) }\end{array}$ \\
\hline Susceptible (50) & 46 & 1 & - & 2 & 1 \\
\hline MDR-TB (100) & 42 & 9 & 34 & 11 & 4 \\
\hline
\end{tabular}

S; susceptible, R; resistant, PZase; pyrazinamidase assay, MGIT; BACTEC MGIT 960 method, pos; positive, neg; negative, wt; wild-type, mut; mutant 
Table 2 Results of pncA gene sequencing of $150 \mathrm{M}$. tuberculosis clinical isolates.

\begin{tabular}{|c|c|c|c|c|}
\hline \multirow[t]{2}{*}{ M. tuberculosis strains (no. of isolates) } & \multirow[t]{2}{*}{ MGIT 960} & \multirow[t]{2}{*}{ PZase assay } & \multicolumn{2}{|l|}{ pncA mutation } \\
\hline & & & Nucleotide change & Amino acid change \\
\hline Susceptible (46) & $S$ & + & wild-type & no \\
\hline Susceptible (1) & $\mathrm{S}$ & + & T92G & Ile31Ser \\
\hline Susceptible (2) & $\mathrm{R}$ & + & wild-type & wild-type \\
\hline Susceptible (1) & $\mathrm{R}$ & + & T92C & Ile31Thr \\
\hline MDR-TB (42) & $S$ & + & wild-type & wild-type \\
\hline MDR-TB (9) & $\mathrm{S}$ & + & T92C & Ile31Thr \\
\hline \multirow[t]{23}{*}{ MDR-TB (34) } & $\mathrm{R}$ & - & $A(-11) G(1)$ & no \\
\hline & & & $A(-11) C(1)$ & no \\
\hline & & & T56G (1) & Leu19Arg \\
\hline & & & $\mathrm{T} 80 \mathrm{C}(1)$ & Leu27Pro \\
\hline & & & T92G (2) & Ile31Ser \\
\hline & & & T104C (1) & Leu35Pro \\
\hline & & & T134C (1) & Val45Ala \\
\hline & & & G136T (1) & Ala46Ser \\
\hline & & & T199C (1) & Ser67Pro \\
\hline & & & C211G (8) & His71Asp \\
\hline & & & G215A (1) & Cys72Tyr \\
\hline & & & G222C (1) & Gly74Arg \\
\hline & & & G289A (3) & Gly97Ser \\
\hline & & & C312G (2) & Ser104Arg \\
\hline & & & G364A (1) & Gly122Ser \\
\hline & & & G373T (1) & Val125Phe \\
\hline & & & G379T (1) & Glu 127 Stop \\
\hline & & & G insertion b/w 411-412 (1) & \\
\hline & & & T416G (1) & Val 139 Gly \\
\hline & & & C425T (1) & Thr 142 Met \\
\hline & & & G436A (1) & Ala $146 \mathrm{Thr}$ \\
\hline & & & C520T (1) & Thr 174 lle \\
\hline & & & GG insertion b/w 520-521 (1) & \\
\hline MDR-TB (11) & R & + & wild-type & no \\
\hline \multirow[t]{2}{*}{ MDR-TB (4) } & $\mathrm{R}$ & + & T92C (3) & \|le31Thr \\
\hline & & & T92G (1) & \|le31Ser \\
\hline
\end{tabular}

$75 \%$ and $89.8 \%$ respectively, when compared with the BACTEC MGIT 960 PZA.

\section{Discussion}

Several studies have reported that the prevalence of PZA resistance ranges from $36 \%$ to $54 \%$ [14,28,29]. In Thailand, there is little information on PZA susceptibility. However, two previous studies have reported the initial PZA resistance to be $6 \%$ and $8 \%$, respectively $[18,23]$. In this study, PZA susceptibility testing by BACTEC MGIT 960 PZA revealed 34.6\% (52/150) PZA resistance. More specifically, PZA resistance was found in $6 \%$ (3/50) of pan-susceptible isolates and 49\% (49/100) of MDR-TB isolates. The results were correlated with those obtained from South Africa indicating 53.3\% (68/127) PZA resistance among previously treated TB patients but a lower resistant rate of $2.1 \%(1 / 47)$ in drug susceptible isolates [14].
PZA resistance is usually associated with defects in PZase activity. Several studies attempted to detect enzyme activity and utilised susceptibility testing for PZA $[18,19,21,22]$. The sensitivity of the PZase assay ranged from 79-96\%, whereas the specificity was approximately 98\% [20-22]. In this study, PZase activity was detected in all 98 PZA-susceptible $M$. tuberculosis isolates but in only 18 of 52 PZA-resistant isolates. Eighteen isolates with positive PZase activity presented discordant results with the MGIT 960 PZA system, resulting in a sensitivity and specificity of $65.4 \%$ and $100 \%$ for that assay, respectively. The sensitivity of our PZase assay is low relative to earlier studies. This might be the result of geographic differences among $M$. tuberculosis isolates. Our isolates may have had alternative resistant mechanisms, such as insufficient drug uptake or the presence of an active efflux pump [10], which 
limits the utility of this test. However, the PZase assay was still useful for screening PZA-resistant $M$. tuberculosis isolates and could be used as an alternative method, particularly for low-income countries where the assay was highly sensitive.

The major mechanism of PZA resistance was associated with mutations of the gene coding for pyrazinamidase, $p n c A$, in which mutations were scattered along the coding and promoter regions with high diversity [7]. In this study, mutations were found in 49 isolates, of which 39 were PZA-resistant and 10 were PZA-susceptible. However, 17 isolates (7 PZA-resistant and 10 PZAsusceptible isolates) showed either Ile31Ser or Ile31Thr mutations. Of these, 15 isolates (except 2 PZA-resistant isolates) had PZase activity. Previous studies have demonstrated the catalytic residues of $M$. tuberculosis PZase that comprise the active (Asp-8, Trp-68, Lys-96, Ser-104, Ala-134, Thr-135 and Cys-138) and metalbinding sites (Asp-49, His-51 and His-71) [30-32]. Taken together with our results, the mutation at Ile-31 did not appear to be associated with PZA resistance. Notably, two PZA-resistant isolates harboured the Ile31Ser mutant but possessed no PZase activity. One possible scenario is that these 2 isolates might have PZase activity that is below the limit of detection for the PZase assay.

Twenty-two of 24 mutation types were detected in this study and showed a correlation with PZA resistance (Table 2). Of these, 14 nucleotide substitutions $[13,14,29,33-36]$ and 2 putative promoter region $[9,33]$ mutations were previously reported. There were 6 novel mutation types, consisting of 3 nucleotide substitutions (Leu27Pro, Gly122Ser, and Thr174Ile), 2 nucleotide insertions ( $G$ insertion between nucleotide 411 and 412 and GG insertion between nucleotide 520 and 521), and 1 nonsense mutation at Glu127. In agreement with earlier studies, the mutations were diverse and scattered throughout the gene sequence, with the most frequently occurring mutation being His71Asp $(8 / 49=16 \%)$. This is not surprising, as His71 is located in one of the three preferably mutated regions (positions 3 to 17,61 to 76 , and 132 to 142) [37] and in the metal-binding site. In addition, there were 13 PZA-resistant isolates (25\%) with observed PZase activity and no mutations in $p n c A$, implying that other unknown mechanisms are involved in PZA resistance.

\section{Conclusions}

This study showed the prevalence of PZA resistance in pan-susceptible and MDR-TB M. tuberculosis clinical isolates from Siriraj Hospital, Thailand. MDR-TB isolates had a much higher percentage of PZA resistance (49\%) than susceptible isolates (6\%). In this study, the sensitivities of the PZase assay and $p n c A$ sequencing were $65 \%$ and $75 \%$, respectively. The results revealed that $25 \%$ of PZA-resistant isolates had wild-type $p n c A$, indicating that phenotypic susceptibility testing was still necessary. Additionally, some pncA mutations are not likely to produce resistance, limiting the use of genotypic methods for PZA susceptibility testing. This information is useful for clinicians in choosing suitable drug regimens for treating TB patients. This study also indicated that the automatic addition of PZA in the treatment regimen of MDR-TB patients would have less benefit in Thailand and would increase the risk of XDR-TB development or render treatment ineffective. Therefore, PZA susceptibility testing in MDR-TB patients should be performed before starting or adjusting treatment regimens.

\section{Acknowledgements}

We would like to thank the Molecular Mycology and Mycobacteriology Laboratory, Drug Resistant Tuberculosis Research Fund, Siriraj Foundation, under the Patronage to Pass HRH Princess Galyani Vadhana Krom Luang Naradhiwas Rajanagarindra, Department of Microbiology, Faculty of Medicine Siriraj Hospital for supporting essential facilities in pyrazinamide susceptibility by the BACTEC MGIT 960 PZA system and all staff members for their help. J was financially supported by the Siriraj Graduate Scholarship. AC was supported by the Chalermphrakiat Grant, Faculty of Medicine Siriraj Hospital, Mahidol University. The study was funded by the Siriraj Graduate Thesis Scholarship, Siriraj Grant for Research and Development, and Drug Resistant Tuberculosis Fund, Siriraj Foundation, Department of Microbiology, Faculty of Medicine Siriraj Hospital. The study was approved by the Siriraj Ethics Committee, Mahidol University. None of the authors has any conflicts of interest to declare.

\section{Author details}

'Department of Microbiology, Faculty of Medicine Siriraj Hospital, Mahidol University, Bangkok, 10700, Thailand. ${ }^{2}$ National Center for Genetic Engineering and Biotechnology, National Science and Technology, Development Agency, Ministry of Science and Technology, Pathumthani 12120, Thailand. ${ }^{3}$ Vichaiyut Hospital, Setsiri Road, Bangkok 10400, Thailand. ${ }^{4}$ Drug-Resistant Tuberculosis Research Fund, Siriraj Foundation, Bangkok 10700, Thailand.

\section{Authors' contributions}

JJ carried out all experiments and drafted the manuscript. TP conceived of the study, participated in its design, performed data analysis and interpretation and helped to draft the manuscript. ML helped to revise the manuscript. AC conceived of the study, participated in its design, helped to critically revise the manuscript and gave final approval of the manuscript. All authors read and approved the final manuscript.

Received: 1 April 2010 Accepted: 20 August 2010

Published: 20 August 2010

\section{References}

1. World Health Organization: WHO Report. Geneva. 2009.

2. Vermund $\mathrm{SH}$, Yamamoto $\mathrm{N}$ : Co-infection with human immunodeficiency virus and tuberculosis in Asia. Tuberculosis (Edinb) 2007, 87(Suppl 1): S18-25.

3. Verma JK, Nateniyom S, Akksilp S, Mankatittham W, Sirinak C, Sattayawuthipong W, Burapat C, Kittikraisak W, Monkongdee P, Cain KP, Wells CD, Tappero JW: HIV care and treatment factors associated with improved survival TB treatment in Thailand: an observational study. BMC Infect Dis 2009, 9:42-50.

4. Cain KP, Anekthananon T, Burapat C, Akksilp S, Mankhatitham W, Sirinak C, Nateniyom S, Sattayawuthipong W, Tasaneeyapan T, Varma JK: Cause of death in HIV-infected persons who have tuberculosis, Thailand. Emerg Infect Dis 2009, 15:258-264. 
5. Mankatittham W, Likanonsakul S, Thawornwan U, Kongsanan $P$, Kittikraisak W, Burapat C, Akksilp S, Sattayawuthipong W, Srinak C, Nateniyom S, Tasaneeyapan T, Verma JK: Characteristics of HIV-infected tuberculosis patients in Thailand. Southeast Asian J Trop Med Public Health 2009, 40:93-103.

6. Zhang Y, Permar S, Sun Z: Conditions that may affect the results of susceptibility testing of Mycobacterium tuberculosis to pyrazinamide. J Med Microbiol 2002, 51:42-9.

7. Zhang $Y$, Mitchison D: The curious characteristics of pyrazinamide: a review. Int J Tuberc Lung 2003, 7:6-21.

8. Zhang $Y$, Wade MW, Scorpio A, Zhang H, Sun Z: Mode of action of pyrazinamide: disruption of Mycobacterium tuberculosis membrane transport and energetics by pyrazinoic acid. J Antimicrob Chermother 2003, 52:790-795.

9. Scorpio A, Zhang $Y$ : Mutation in $p n c A$, a gene encoding pyrazinamidase/ nicotinamidase, caused resistance to antituberculous drug, pyrazinamide in tubercle bacillus. Nature Med 1996, 2:662-667.

10. Singh P, Mishra AK, Malonia SK, Chauhan DS, Sharma VD, Venkatesan K, Katoch VM: The paradox of pyrazinamide: an update on the molecular mechanisms of pyrazinamide resistance in mycobacteria. J Commun Dis 2006, 38:288-298.

11. Mestdagh M, Fonteyne PA, Realini L, Rossau R, Jannes G, Mijs W, de Smet KAL, Portaels F, Eeckhout VD: Relationship between pyrazinamide resistance, loss of pyrazinamidase activity, and mutations in the pncA locus in multidrug-resistant clinical isolates of Mycobacterium tuberculosis. Antimicrob Agents Chemother 1999, 43:2317-2319.

12. Mphahlele $M$, Syre $H$, Valvatne $H$, Stavrum $R$, Mannsaker $T$, Mothivhi $T$, Weyer K, Fourie PB, Grewal HM: Pyrazinamide resistance among South African multidrug-resistant Mycobacterium tuberculosis isolates. J Clin Microbiol 2008, 46:3459-3464.

13. Cheng SJ, Thibert L, Sanchez T, Heifets $L$, Zhang $Y$ : pncA mutations as a major mechanism of pyrazinamide resistance in Mycobacterium tuberculosis: spread of a monoresistant strain in Quebec, Canada. Antimicrob Agents Chemother 2000, 44:528-532.

14. Louw GE, Warren RM, Donald PR, Murray MB, Bosman M, van Helden PD, Young DB, Victor TC: Frequency and implications of pyrazinamide resistance in managing previously treated tuberculosis patients. Int $J$ Tuberc Lung Dis 2006, 10:802-807.

15. Woods G, Desmond EP, Hall GS, Heifets L, Pfyffer GE: Susceptibility testing of mycobacteria, norcardiae, and other aerobic Actinomycetes: Approved standard NCCLS document M24-A. NCCLS 2003.

16. Scarparo C, Ricardo P, Ruggiero G, Piccoli P: Evaluation of the fully automated BACTEC MGIT 960 system for testing susceptibility of Mycobacterium tuberculosis to pyrazinamide, streptomycin, isoniazid, rifampicin and ethambutol and comparison with the radiometric BACTEC 460 TB method. J Clin Microbiol 2004, 42:1109-1114.

17. Pfyffer GE, Palicova F, Rusch-Gerdes S: Testing of susceptibility of Mycobacterium tuberculosis to pyrazinamide with the nonradiometric BACTEC MGIT 960 system. J Clin Microbiol 2003, 40:1670-1674.

18. Rienthong S, Rienthong D, Smithikarn S, Yamnimnual S: Study of initial drug resistance of pyrazinamide in new pulmonary tuberculosis patients before treatment in tuberculosis division by detection of enzyme pyrazinamidase. Thai J Tuberc Chest Dis 1993, 14:85-89.

19. Miller MA, Thibert $L$, Desjardins F, Siddiqi SH, Dascal A: Testing of susceptibility of Mycobacterium tuberculosis to pyrazinamide: comparison of Bactec method with pyrazinamidase assay. J Clin Microbiol 1995, 33:2468-2470.

20. Davies AP, Billington OJ, McHugh TD, Mitchison DA, Gillespie SH: Comparison of phenotypic and genotypic methods for pyrazinamide susceptibility testing with Mycobacterium tuberculosis. J Clin Microbiol 2000, 38:3686-3688.

21. Krishnamurthy A, Almeida D, Rodrigues C, Mehta A: Comparison of pyrazinamide drug susceptibility of $M$. tuberculosis by radiometric Bactec and enzymatic pyrazinamidase assay. Indian. J Med Microbiol 2004, 22:166-168.

22. Singh P, Wesley $C$, Jadaun GPS, Malonia SK, Das R, Upadhyay P, Faujdar J, Sharma P, Gupta P, Mishra AK, Singh K, Chauhan DS, Sharma VD, Gupta UD, Venkatesan K, Katoch VM: Comparative evaluation of Lowenstein-Jensen proportion method, BacT/ALERT 3 D system, and enzymatic pyrazinamidase assay for pyrazinamide susceptibility testing of Mycobacterium tuberculosis. J Clin Microbiol 2007, 45:76-80.
23. Pransucharit $V$, Chuchottaworn $C$ : Detection of initial drug resistance of pyrazinamide in Thai tuberculosis patients by radiometric method. Thai J Tuberc Chest Dis 1995, 16:59-65.

24. Chaiprasert A, Prammananan T, Tingtoy N, Na-Ubol P, Srimuang S, Samerpitak K, Rangsipanuratn W: One-tube multiplex PCR method for rapid identification of Mycobacterium tuberculosis. Southeast Asian J Trop Med Public Health 2006, 37:494-502.

25. Kent PT, Kubica GP: Plublic health mycobacteriology. A guide for the level III laboratory. Atlanta: US Department of health and human service 1985.

26. Wayne LG: Simple pyrazinamidase and urease tests for routine identification of mycobacteria. Am Rev Respir Dis 1974, 109:147-151.

27. Cheunoy W, Prammananan T, Chaiprasert A, Foongladda S: Comparative evaluation of polymerase chain reaction and restriction enzyme analysis: two amplified targets, $h s p 65$ and $r p o B$ for identification of cultured mycobacteria. Diagn Microbiol Infect Dis 2005, 51:165-171.

28. Hou L, Osei-Hyiaman D, Zhang Z, Wang B, Yang A, Kano K: Molecular characterization of pncA gene mutations in Mycobacterium tuberculosis clinical isolates from China. Epidemiol Infect 2000, 124:227-232.

29. Chan RC, Hui M, Chan EW, Au TK, Chin ML, Yip CK, AuYeang CK, Yeung CY, Kam KM, Yip PC, Cheng AF: Genetic and phenotypic characterization of drug-resistant Mycobacterium tuberculosis isolates in Hong Kong. J Antimicrob Chemother 2007, 59:866-873.

30. Lemaitre N, Sougakoff W, Truffot-Pernot C, Jarlier V: Characterization of new mutations in Pyrazinamide-resistant strains of Mycobacterium tuberculosis and identification of conserved regions important for the catalytic activity of the pyrazinamidase PncA. Antimicrob Agents Chemother 1999, 43:1761-1763.

31. Lemaitre N, Callebaut I, Frenois F, Jarlier V, Sougakoff W: Study of the structure-activity relationships for the pyrazinamidase (PncA) from Mycobacterium tuberculosis. Biochem J 2001, 353:453-458.

32. Du X, Wang W, Kim R, Yakota H, Nguyen H, Kim SH: Crystal structure and mechanism of catalysis of a pyrazinamidase from Pyrococcus horikoshii. Biochemistry 2001, 40:14166-14172.

33. Sreevatsan S, Pan X, Zhang Y, Kreiswirth BN, Musser JM: Mutations associated with pyrazinamide resistance in pncA of Mycobacterium tuberculosis complex organisms. Antimicrob Agents Chemother 1997, 41:636-640.

34. Hirano K, Takahashi M, Kazumi Y, Fukasawa Y, Abe C: Mutation in pncA is a major mechanism of pyrazinamide resistance in Mycobacterium tuberculosis. Tuber Lung Dis 1997, 78:117-122.

35. Morlock GP, Crawford JT, Butler ER, Brim SE, Sikes D, Mazurek GH, Woodley CL, Cooksey RC: Phenotypic characterization of pncA mutants of Mycobacterium tuberculosis. Antimicrob Agents Chemother 2000, 44:2291-2295.

36. Lee KW, Lee JM, Jung KS: Characterization of pncA mutations pyrazinamide- resistant Mycobacterium tuberculosis in Korea. J Korean Med Sci 2001, 16:537-543.

37. Scorpio A, Lindholm-Levy P, Heifets L, Gilman R, Siddiqi S, Cynamon M, Zhang Y: Characterization of pncA mutations in pyrazinamide-resistance Mycobacterium tuberculosis. Antimicrob Agents Chemother 1997, 41:540-543.

doi:10.1186/1471-2180-10-223

Cite this article as: Jonmalung et al:: Surveillance of pyrazinamide susceptibility among multidrug-resistant Mycobacterium tuberculosis isolates from Siriraj Hospital, Thailand. BMC Microbiology 2010 10:223. 